\title{
Awarded Processing Technique
}

What does the cover photo of this magazine have to do with the Nobel Prize?

Glass, an important material in the ceramics family, has become an integral part of everyday life. With growing digitization, increasing networking and communication, this material is becoming more and more important and is taking on a whole new meaning. Glass plays a very special role in screens, touch displays, sensors, camera modules, micro-batteries and chip packaging, to name just a few examples. Numerous technological innovations have given the material completely new properties. New areas of application become possible, new markets emerge and processing techniques must be adapted and work with high-precision.

The so-called ultra-short pulse laser is suitable for the efficient, ultra-precise cutting of particularly fine and sensitive materials such as glass. Two of this year's Nobel Prize winners in physics, Gérard Mourou and Donna Strickland, were awarded for the method of generating ultrashort laser pulses of high intensity, today an important standard technology in research, industry and medical applications, e.g. for eye surgeries. Chirped Pulse Amplification technology has made it possible to gently process a wide variety of materials with very short and at the same time extremely powerful laser pulses.

Prof. Dr. Gérard Mourou has received numerous prizes for his work, including the Berthold Leibinger Research Award for Applied Laser Technology in Germany in 2016. The scientist Mourou is considered a pioneer and one of the most creative and visionary minds in the field of laser physics. He initiated the establishment of high-performance laser centers worldwide, most recently the European large-scale project Extreme Light Infrastructure (ELI). In addition, the passionate networker forged an international community of scientists in this field.

The photo on the front page shows an ultra-short pulsed laser in industrial use for processing displays of mobile phones. We are pleased, that our ceramics industry can also benefit from this technology, and congratulate! 4 